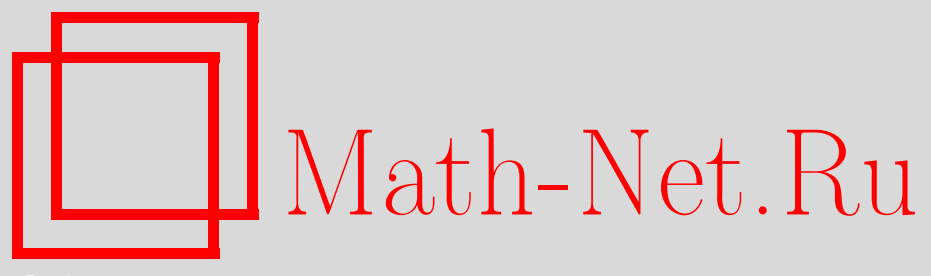

П. Н. Вабищевич, Сходимость алгоритма типа Метрополиса для большого канонического ансамбля, Матем. заметки, 2007, том 82, выпуск 4, 519-524

DOI: https://doi.org/10.4213/mzm3822

Использование Общероссийского математического портала Math-Net.Ru подразумевает, что вы прочитали и согласны с пользовательским соглашением http://www . mathnet.ru/rus/agreement

Параметры загрузки:

IP: 18.234 .197 .8

26 апреля 2023 г., 13:00:19

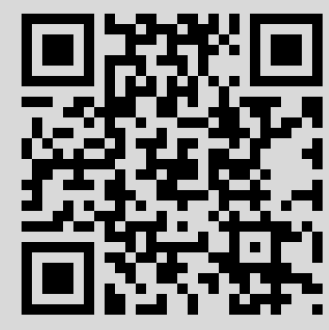


Том 82 выпуск 4 октябрь 2007

УДК 519.217

\section{Сходимость алгоритма типа Метрополиса для большого канонического ансамбля}

\section{Н. П. Вабищевич}

В работе исследуется вопрос сходимости алгоритмов типа Метрополиса, использующихся при моделировании статистических систем с переменным числом частиц, находящихся в ограниченном объеме. В работе обосновано использование алгоритмов Метрополиса для определенного класса таких статистических систем. Доказана теорема о геометрической эргодичности марковского процесса, моделирующего поведение ансамбля переменного числа частиц в ограниченном объеме, взаимодействие которых описывается ограниченным снизу потенциалом, растущим как $r^{-3-\alpha}, \alpha \geqslant 0$, при $r \rightarrow 0$.

Библиография: 6 названий.

1. Алгоритмы типа Метрополиса-Хастингса. Для численного нахождения различных средних по статистическим ансамблям используются разновидности алгоритма Метрополиса-Хастингса [1], состоящего в следующем.

Пусть дана ненормированная функция плотности $\pi(\cdot)$ на пространстве $\mathscr{X}$, которой можно сопоставить вероятностную меру

$$
\tilde{\pi}(A)=\frac{\int_{A} \pi(x) d x}{\int_{\mathscr{X}} \pi(x) d x} .
$$

Предположим, что необходимо оценить среднее функции $f: \mathscr{X} \rightarrow \mathbb{R}$ по распределению $\pi(A)$, т.е. необходимо оценить величину

$$
\tilde{\pi}(f)=\frac{\int_{\mathscr{X}} f(x) \pi(x) d x}{\int_{\mathscr{X}} \pi(x) d x} .
$$

Возьмем некоторый “затравочный” марковский процесс с переходной вероятностью $Q(x, d y) \propto q(x, y) d y$, допускающий эффективную численную реализацию. От выбора этого процесса зависит характерное время сходимости результирующего процесса Метрополиса-Хастингса $(\mathrm{M}-\mathrm{X})$ к стационарному распределению. Процесс $\mathrm{M}-\mathrm{X}$ со стационарным распределением $\widetilde{\pi}(\cdot)$ строится следующим образом. Имея $X_{n}$ и $Q$, строим $Y_{n+1}$ :

$$
\mathbb{P}\left\{Y_{n+1} \in A \mid X_{n}\right\}=\int_{A} Q\left(X_{n}, d y\right) .
$$

Затем, с вероятностью $\alpha\left(X_{n}, Y_{n+1}\right)$, где

$$
\alpha(x, y)=\min \left[1, \frac{\pi(y) q(y, x)}{\pi(x) q(x, y)}\right],
$$

(C) Н. П. ВАБищЕвич, 2007 
принимаем $X_{n+1}=Y_{n+1}$ или с вероятностью $1-\alpha\left(X_{n}, Y_{n+1}\right)$ оставляем $X_{n+1}=X_{n}$. Используемая конструкция является функциональным аналогом метода отбора Неймана,

$$
\mathbb{P}\left\{X_{n+1} \in A \mid X_{n}\right\}=\int_{A} \alpha\left(X_{n}, y\right) Q\left(X_{n}, d y\right)+\mathbf{1}_{A}\left(X_{n}\right) \int_{\mathscr{X}}\left[1-\alpha\left(X_{n}, y\right)\right] Q\left(X_{n}, d y\right) .
$$

В случае $q(x, y)=q(y, x)$ получаем симметричный алгоритм Метрополиса [2], в котором

$$
\alpha(x, y)=\min \left[1, \frac{\pi(y)}{\pi(x)}\right]
$$

Если две цепочки Маркова с переходными операторами $P_{1}$ и $P_{2}$ имеют одно и то же стационарное распределение $\widetilde{\pi}(\cdot)$ (не обязательно единственное), то распределение $\tilde{\pi}(\cdot)$ также является стационарным для цепочки с оператором $P_{1} P_{2}$. На этом наблюдении основаны способы комбинирования процессов Метрополиса.

Пусть дано $d$-мерное распределение $\widetilde{\pi}(\cdot)$ на $\mathscr{X}=\mathscr{Y}^{d}$. Зададим оператор перехода $P_{i}$, который оставляет все компоненты, кроме $i$-й, неизменными, а $i$-ю заменяет компонентой соответствующей переходу по алгоритму Метрополиса для условного распределения с фиксированными компонентами $\{1,2, \ldots, i-1, i+1, \ldots, d\}$. Тогда цепь Маркова с оператором перехода

$$
P=P_{1} P_{2} \cdots P_{d}
$$

будет иметь $d$-мерное стационарное распределение $\widetilde{\pi}(\cdot)$. Также можно использовать алгоритм равновероятного случайного выбора:

$$
P=\frac{1}{d}\left(P_{1}+P_{2}+\cdots+P_{d}\right)
$$

Одним из свойств цепей Маркова, характеризующих их сходимость, является геометрическая эргодичность [3].

Цепь Маркова со стационарным распределением $\widetilde{\pi}(\cdot)$ называется геометрически эргодичной, если

$$
\left\|P^{n}(x, \cdot)-\pi(\cdot)\right\| \leqslant M(x) \rho^{n}, \quad n=1,2,3, \ldots,
$$

для некоторого $\rho<1$, где $M(x)<\infty \widetilde{\pi}$-п.в. для $x \in \mathscr{X}$.

Для проверки эргодичности цепи используется условие дрейфа [3], [4].

Множество $C$ называется малым, если существует целое положительное число $n$, $\varepsilon>0$ и вероятностная мера $\nu(\cdot)$ такие, что

$$
P^{n}(x, \cdot) \geqslant \varepsilon \nu(\cdot) \quad x \in C .
$$

Цепь Маркова удовлетворяет условию дрейфа в направлении множества $C$, если существуют константы $0<\lambda<1$ и $b<\infty$, а также функция $V: \mathscr{X} \rightarrow[1, \infty]$ такие, что

$$
P V \leqslant \lambda V+b \mathbf{1}_{C} .
$$


В работе [4] приводится следующее важное утверждение.

Теорема 1. Если ч-неприводимая апериодическая иепь Маркова со стационарным распределением $\pi(\cdot)$ удовлетворяет условию дрейфа в направлении малого множества $C$ с $V(x)<\infty$ хотя бы для одного $x$, то эта цепь является геометрически эргодичной.

В дальнейшем изложении мы используем данный факт. Напомним [3]-[5], что цепь Маркова является $\varphi$-неприводимой, если существует мера $\varphi$ на $\mathscr{X}$ такая, что для любого $A \subseteq \mathscr{X}$, для которого $\varphi(A)>0$, и для всех $x \in \mathscr{X}$ существует положительное целое $n=n(x, A)$ такое, что $P^{n}(x, A)>0$.

\section{2. Алгоритмы типа Метрополиса для большого канонического ансам-}

бля. Рассмотрим термодинамическую систему с переменным числом частиц в ограниченном объеме $\mathbb{V}$ (сюда также относится случай периодических граничных условий). Функция плотности в этом случае имеет вид $\pi=e^{-S}$ (см. [6]), где

$$
S=\frac{E-\mu N}{T}+N\left[\ln \left(N \Lambda^{3}\right)-1\right],
$$

заданный в пространстве $\mathscr{X}=\left\{\mathbf{r}_{1}, \mathbf{r}_{2}, \ldots, \mathbf{r}_{N}\right\}_{N=0}^{\infty} ; T$ и $\mu$ здесь - внешние параметры, $\Lambda=2 \pi \hbar / \sqrt{2 m T}$, а $E$ - энергия системы:

$$
E=\sum_{i=1}^{N} U\left(\mathbf{r}_{i}\right)+\sum_{1 \leqslant i<j \leqslant N} \varphi\left(\left|\mathbf{r}_{i}-\mathbf{r}_{j}\right|\right)
$$

При изучении таких систем используются два типа переходов: с изменением состояния (например, переносом) и с рождением или уничтожением одной частицы. Обозначим их $P_{i}$ и $P_{ \pm}$соответственно. Переход $P_{i}$ соответствует виртуальному выбору нового положения $i$-й частицы (процесс $Q$ ), и его реализации с вероятностью, используемой в алгоритме Метрополиса:

$$
\alpha=\min \left(1, \exp \left\{\frac{E-\left.E\right|_{\mathbf{r}_{i}=\mathbf{r}_{\text {new }}}}{T}\right\}\right)=\min \left(1, \exp \left\{\frac{E_{i}-\left.E_{i}\right|_{\mathbf{r}_{i}=\mathbf{r}_{\text {new }}}}{T}\right\}\right)
$$

где

$$
E_{i}=U\left(\mathbf{r}_{i}\right)+\sum_{\substack{1 \leqslant j \leqslant N \\ j \neq i}} \varphi\left(\left|\mathbf{r}_{i}-\mathbf{r}_{j}\right|\right)
$$

Такое правило реализует переход в новую точку с вероятностью единица, если энергия системы уменьшается, и с экспоненциально малой вероятностью, если энергия системы увеличивается. Более того, при увеличении температуры строгость этого правила уменьшается.

Переход $P_{ \pm}=\left(P_{+}+P_{-}\right) / 2$ состоит в равновероятном выборе рождения или уничтожения. Для $P_{+}$производится случайный выбор нового положения из объема $\mathbb{V}$ и его принятие с вероятностью

$$
\alpha=\min \left(1, \frac{\mathbb{V}}{(N+1) \Lambda^{3}} \exp \left\{-\frac{E_{\text {new }}-\mu}{T}\right\}\right),
$$


где

$$
E_{\text {new }}=U\left(\mathbf{r}_{\text {new }}\right)+\sum_{i=1}^{N} \varphi\left(\left|\mathbf{r}_{i}-\mathbf{r}_{\text {new }}\right|\right) .
$$

Для $P_{-}$равновероятно выбирается одна из $N$ частиц и уничтожается с вероятностью

$$
\alpha=\min \left(1, \frac{N \Lambda^{3}}{\mathbb{V}} \exp \left\{\frac{E_{\mathrm{del}}-\mu}{T}\right\}\right),
$$

где

$$
E_{\mathrm{del}}=U\left(\mathbf{r}_{\mathrm{del}}\right)+\sum_{\substack{1 \leqslant i \leqslant N \\ i \neq i \mathrm{del}}} \varphi\left(\left|\mathbf{r}_{i}-\mathbf{r}_{\mathrm{del}}\right|\right) .
$$

Эти переходы тем или иным образом комбинируются. В качестве примера можно предложить случайный выбор между типами переходов:

$$
P=q \frac{P_{+}+P_{-}}{2}+(1-q) \frac{1}{N} \sum_{i=1}^{N} P_{i},
$$

где $N$ - количество частиц перед началом перехода; или одно изменение числа с последующим перемещением $n$ случайно выбранных частиц:

$$
P=\frac{P_{+}+P_{-}}{2}\left[\frac{1}{N} \sum_{i=1}^{N} P_{i}\right]^{n},
$$

где $N$ - количество частиц после изменения числа.

Наложим следующие ограничения на потенциалы:

1) потенциальная энергия частиц во внешнем поле ограничена снизу (в данном случае без ограничения общности будем считать ее неотрицательной, $U(\mathbf{r}) \geqslant 0)$;

2) энергия парных взаимодействий удовлетворяет условию $\varphi(r) \geqslant \varphi_{0}+C r^{-\gamma}$, где $\varphi_{0}<0, C>0$ и $\gamma>3$.

В этом случае ниже мы установим геометрическую эргодичность алгоритма. Для начала докажем следующую лемму.

Лемма 1. Если $P(x, \cdot)$ - вероятность перехода для процесса типа Meтрополиса с ненормированной плотностъю $\pi$ и затравочной переходной вероятностъю $Q(x, \cdot), V=\pi^{-\alpha}$, где $0<\alpha<1$, то справедливы оченки

$$
\begin{aligned}
& \int P(x, d y) V(y) \leqslant V(x) \int Q(x, d y) \frac{\pi(x)^{\alpha}}{\pi(y)^{\alpha}}, \\
& \int P(x, d y) V(y) \leqslant V(x)\left[1+\alpha(1-\alpha)^{1 / \alpha-1}\right] .
\end{aligned}
$$

ДокАЗАТЕЛЬСтво. В силу сделанных предположений

$$
\begin{gathered}
\int P(x, d y) V(y)=\int_{\pi(y) \geqslant \pi(x)} Q(x, d y) V(y)+\int_{\pi(y)<\pi(x)} Q(x, d y) \frac{\pi(y)}{\pi(x)} V(y) \\
+V(x) \int_{\pi(y)<\pi(x)} Q(x, d y)\left(1-\frac{\pi(y)}{\pi(x)}\right)
\end{gathered}
$$




$$
\begin{aligned}
& =V(x) \int_{\pi(y) \geqslant \pi(x)} Q(x, d y) \frac{\pi(x)^{\alpha}}{\pi(y)^{\alpha}}+V(x) \int_{\pi(y)<\pi(x)} Q(x, d y) \frac{\pi(y)^{1-\alpha}}{\pi(x)^{1-\alpha}} \\
& \quad+V(x) \int_{\pi(y)<\pi(x)} Q(x, d y)\left(1-\frac{\pi(y)}{\pi(x)}\right) \\
& =V(x) \int Q(x, d y) F\left(\frac{\pi(y)}{\pi(x)}\right)
\end{aligned}
$$

где

$$
F(w)= \begin{cases}1+w^{1-\alpha}-w, & \text { если } w<1 \\ w^{-\alpha}, & \text { если } w \geqslant 1\end{cases}
$$

Так как при всех $w \in(0,1]$ имеет место следующее неравенство:

$$
\frac{d}{d w}\left[\left(1+w^{1-\alpha}-w\right)-w^{-\alpha}\right]=(1-\alpha) w^{-\alpha}+\alpha w^{-1-\alpha}-1>0,
$$

то $F(w) \leqslant w^{-\alpha}$ и оценка $(2)$ справедлива. Найдем максимум функции $F$. Поскольку

$$
\frac{d}{d w}\left[1+w^{1-\alpha}-w\right]=(1-\alpha) w^{-\alpha}-1,
$$

то $w_{\max }=(1-\alpha)^{1 / \alpha}$ и потому

$$
F_{\max }=F\left[(1-\alpha)^{1 / \alpha}\right]=1+(1-\alpha)^{1 / \alpha-1}-(1-\alpha)^{1 / \alpha}=1+\alpha(1-\alpha)^{1 / \alpha-1} .
$$

Следовательно, верна и оценка (3).

Для проверки достаточного условия эргодичности из теоремы 1 используем условие дрейфа с $V=\pi^{-\alpha}$ при некотором $0<\alpha<1$ в направлении множества состояний с числом частиц не больше, чем $N_{0}$, для достаточно большого $N_{0}$.

Для функций $P_{i} V$ и $P_{+} V$ используем оценку сверху величиной $V\left[1+\alpha(1-\alpha)^{1 / \alpha-1}\right]$, которая выбором $\alpha$ близким к 1 может быть сделана сколь угодно близкой к $V$. Если при этом $P_{-} V<\lambda V$ с постоянной $\lambda<1$, не зависящей от $\alpha$, то подходящим выбором $\alpha$ можно удовлетворить условию дрейфа (1) в случае, если на каждый переход, соответствующий оператору $P_{ \pm}$, приходится ограниченное сверху количество переходов, соответствующих $P_{i}$.

Перейдем к рассмотрению процесса $P_{-}$- процесса уничтожения частиц. Назовем "обособленными" те частицы, энергия $E_{i}$ которых меньше некоторого $E_{0}$. Конкретное значение $E_{i}$ выберем позже. Согласно предположениям

$$
E_{i} \geqslant(N-1) \varphi_{0}+C\left|\mathbf{r}_{j}-\mathbf{r}_{i}\right|^{-\gamma} \quad \forall j \neq i,
$$

поэтому расстояние от “обособленной” частицы до любой другой больше, чем

$$
R=\left[\frac{C}{E_{0}-(N-1) \varphi_{0}}\right]^{1 / \gamma}
$$

Поскольку полный объем $\mathbb{V}$ ограничен, то количество обособленных частиц меньше, чем

$$
\frac{\mathbb{V}}{\pi R^{3} / 6}=\frac{6 \mathbb{V}}{\pi}\left[\frac{E_{0}-(N-1) \varphi_{0}}{C}\right]^{3 / \gamma}<\frac{6 \mathbb{V}}{\pi}\left[\frac{E_{0}-\left(N_{0}-1\right) \varphi_{0}}{C}\right]^{3 / \gamma} \equiv N_{\text {sol }}
$$


при $N>N_{0}$. Теперь можно сделать следующую оценку:

$$
\begin{aligned}
P_{-} V & \leqslant \frac{N_{\text {sol }}}{N}\left[1+\alpha(1-\alpha)^{1 / \alpha-1}\right] V+\frac{N-N_{\text {sol }}}{N}\left[\frac{N \Lambda^{3}}{\mathbb{V}} \exp \left\{\frac{E_{0}-\mu}{T}\right\}\right]^{-\alpha} V \\
& <\frac{N_{\text {sol }}}{N_{0}}\left[1+\alpha(1-\alpha)^{1 / \alpha-1}\right] V+\left[\frac{N_{0} \Lambda^{3}}{\mathbb{V}} \exp \left\{\frac{E_{0}-\mu}{T}\right\}\right]^{-\alpha} V .
\end{aligned}
$$

Если $\gamma>3$, то $N_{\text {sol }}$ растет медленнее, чем $N_{0}$, и выбором $N_{0}$ первое слагаемое можно сделать сколь угодно малым. Аналогично, при любом $\alpha$ выбором $E_{0}$ можно сделать малым второе слагаемое.

Таким образом, доказана следующая

Теорема 2. Марковский прочесс, построенный из операторов $P_{i}$ и $P_{ \pm}$, в котором на каждый оператор $P_{ \pm}$приходится ограниченное сверху количество операторов $P_{i}$, геометрически эргодичен.

3. Выводы. Из геометрической эргодичности цепи Маркова следует справедливость теорем типа ЦПТ для выборочных средних от наблюдаемых с конечным $(2+\delta)$-м моментом $(\delta>0)$, что обосновывает использование таких алгоритмов при численном моделировании систем описанного класса.

\section{СПИСОК ЦИТИРОВАННОЙ ЛИТЕРАТУРЫ}

[1] W. K. Hastings, "Monte-Carlo sampling methods using Markov chains and their applications", Biometrika, 57:1 (1970), 97-109.

[2] N. Metropolis, A.W. Rosenbluth, M. N. Rosenbluth, A.H. Teller, "Equations of state calculations by fast computing machines", J. Chem. Phys., 21:6 (1953), 1087-1092.

[3] S. P. Meyn, R. L. Tweedie, Markov chains and stochastic stability, Communications and Control Engineering Series, Springer-Verlag, London, 1993.

[4] G. O. Roberts, J.S. Rosenthal, General state space Markov chains and MCMC algorithms, arXiv: math/0404033.

[5] K. L. Mengersen, R. L. Tweedie, "Rates of convergence of the Hastings and Metropolis algorithms", Ann. Statist., 24:1 (1996), 101-121.

[6] Л. Д. Ландау, Е. М. Лифшиц, Теоретическая физика, т. 5: Статистическая физика, Наука, М., 1964.

\section{Н. П. Вабищевич}

Поступило

Московский государственный университет

12.03.2007

им. М. В. Ломоносова

E-mail: npvab@list.ru 